\title{
Dyslipidemia and Blood-Brain Barrier Integrity in Alzheimer's Disease
}

\author{
Gene L. Bowman, ${ }^{1}$ Jeffrey A. Kaye, ${ }^{1,2,3}$ and Joseph F. Quinn ${ }^{1,3}$ \\ ${ }^{1}$ Department of Neurology, Oregon Health and Science University, 3181 Southwest Samuel Jackson Park Road, \\ Portland, OR 97239, USA \\ ${ }^{2}$ Biomedical Engineering, Oregon Health and Science University, Portland, OR 97239, USA \\ ${ }^{3}$ The Portland Veteran Affairs Medical Center, Portland, OR, USA
}

Correspondence should be addressed to Gene L. Bowman, bowmang@ohsu.edu

Received 21 December 2011; Accepted 27 January 2012

Academic Editor: Andrea Fuso

Copyright (C) 2012 Gene L. Bowman et al. This is an open access article distributed under the Creative Commons Attribution License, which permits unrestricted use, distribution, and reproduction in any medium, provided the original work is properly cited.

\begin{abstract}
Background. Blood-brain barrier (BBB) dysfunction may have a significant role in the pathogenesis of Alzheimer's disease (AD). Modifiable factors associated with BBB function may have therapeutic implication. This study tested the hypothesis that dyslipidemia is associated with BBB impairment in mild-to-moderate AD. Methods. Thirty-six subjects with AD were followed for 1 year. Fasting CSF and plasma were collected with clinical assessments at baseline and 12 months. BBB impairment was defined as CSF albumin index $\geq 9$. Independent $t$-tests and linear regression assessed the relationship between plasma lipoproteins and BBB integrity. Results. Dyslipidemia was prevalent in $47 \%$ of the population, and in $75 \%$ of those with BBB impairment. Subjects with $\mathrm{BBB}$ impairment had significantly higher mean plasma triglyceride and lower HDL cholesterol (TG, $P=0.007$; HDL, $P=0.043$ ). Plasma triglycerides explained $22 \%$ of the variance in BBB integrity and remained significant after controlling for age, gender, ApoE-4 genotype, blood pressure, and statin use. Conclusion. Dyslipidemia is more prevalent in AD subjects with BBB impairment. Plasma triglyceride and HDL cholesterol may have a role in maintaining BBB integrity in mild-to-moderate Alzheimer's disease.
\end{abstract}

\section{Introduction}

The CSF albumin index is an established measure of bloodbrain barrier (BBB) integrity in living patients [1]. This index has detected a higher prevalence of BBB impairment in late onset dementia, including both Alzheimer's disease (AD) and vascular dementia compared to cognitively intact elders [2]. BBB impairment is associated with more rapid rate of decline in AD over 1 year [3]. Capillary endothelia dysfunction and the approximate tight junctions between these cells may be important to $\mathrm{AD}$ pathogenesis, including effects on maintaining cerebral perfusion and the clearance of toxic forms of beta-amyloid protein $[4,5]$. The notion that the $\mathrm{BBB}$ is central to the pathogenesis of $\mathrm{AD}$ is controversial, but the enormity of the cerebrovascular tree and the similarity in risk factors between vascular and Alzheimer's disease makes the $\mathrm{BBB}$ and neurovascular unit difficult to disregard $[6,7]$.
If $\mathrm{BBB}$ function plays a role in the pathogenesis of $\mathrm{AD}$, then modifiable factors associated with it may have therapeutic potential. A clinical trial of B vitamin supplementation has suggested that the BBB may be a modifiable entity in subjects with hyperhomocysteinemia and mild cognitive impairment [8]. Lipids are plausible candidates for modifying BBB function because of their relationship with vascular disease and ability to affect $\mathrm{AD}$ type pathology [9]. This study examines the relationship between dyslipidemia and BBB integrity in a well-characterized sample with mild-tomoderate Alzheimer's disease.

\section{Methods}

2.1. Study Population. The study participants were recruited from the clinic population of the NIA-Layton Aging and Alzheimer's Disease Center of Oregon Health and Science 
TABLE 1: Study population characteristics ${ }^{1}$.

\begin{tabular}{|c|c|c|c|}
\hline & Total $(n=36)$ & BBB intact $(n=28)$ & BBB impaired $(n=8)$ \\
\hline Age, years & $70(7.1)$ & $70(7)$ & $73(8)$ \\
\hline Female, no. $(\%)$ & $12(33)$ & $11(39)$ & $1(13)$ \\
\hline ApoE-e4 allele carriers (\%) & $27(75)$ & $21(75)$ & $6(75)$ \\
\hline BMI $(\%)$ & $27(4.6)$ & $26(4)$ & $29(6)$ \\
\hline BP, systole, mmHg & $142(23.2)$ & $143(21)$ & $137(30)$ \\
\hline $\mathrm{BP}$, diastole, $\mathrm{mmHg}$ & $78(12.2)$ & $79(13)$ & $76(8)$ \\
\hline Mini mental state exam & $19(5.0)$ & $20(5)$ & $18(5)$ \\
\hline Hachinski ischemia score & $0.6(0.9)$ & $0.5(0.8)$ & $1.0(1.3)$ \\
\hline Statin use, no. (\%) & $8(22)$ & $5(18)$ & $3(38)$ \\
\hline Glucose, mg/dL & $99(18)$ & $98(18)$ & $101(17)$ \\
\hline CSF albumin index & $7.2(3.7)$ & $5.6(1.7)$ & $12.9(3.3)^{\mathrm{abc}}$ \\
\hline Atherogenic dyslipidemia, no. $(\%)^{2}$ & $15(42)$ & $9(32)$ & $6(75)^{\mathrm{a}}$ \\
\hline Metabolic dyslipidemia, no. $(\%)^{2}$ & $17(47)$ & $11(39)$ & $6(75)^{\mathrm{a}}$ \\
\hline Triglycerides, mg/dL & $215(132)$ & $185(86)$ & $323(204)^{\mathrm{ab}}$ \\
\hline HDL cholesterol, mg/dL & $48(20)$ & $52(22)$ & $35(5)^{\mathrm{a}}$ \\
\hline LDL cholesterol, mg/dL & $129(33)$ & $132(37)$ & $120(10)$ \\
\hline Total cholesterol, mg/dL & $216(36)$ & $218(40)$ & $210(27)$ \\
\hline
\end{tabular}

BBB: blood-brain barrier, ApoE-e4: apolipoprotein E epsilon 4, BMI: body mass index, BP: blood pressure, HDL: high-density lipoprotein, LDL: low-density lipoprotein.

${ }^{1}$ Mean and standard deviation provided unless stated otherwise; ${ }^{\mathrm{a}} \mathrm{P}<0.05,{ }^{\mathrm{ab}} \mathrm{P}<0.01,{ }^{\mathrm{abc}} \mathrm{P}<0.001$.

${ }^{2}$ Atherogenic dyslipidemia: triglycerides $\geq 150 \mathrm{mg} / \mathrm{dL}, \mathrm{LDL}>100 \mathrm{mg} / \mathrm{dL}$ and $\mathrm{HDL}<50 \mathrm{mg} / \mathrm{dL}[15]$.

${ }^{3}$ Metabolic dyslipidemia: triglycerides $\geq 150 \mathrm{mg} / \mathrm{dL}, \mathrm{HDL}<50 \mathrm{mg} / \mathrm{dL}[16]$.

University and have been described previously [3]. Briefly, all subjects had a consensus diagnosis of probable $\mathrm{AD}$ using the National Institute of Neurological and Communicative Disorders and Stroke/Alzheimer's Disease and Related Disorders Association criteria and a Clinical Dementia Rating of 0.5 or 1 to establish disease stage of mild-to-moderate AD. All participants provided informed consent in accord with the Institutional Review Board for human study at Oregon Health and Science University. All subjects had CSF and blood available for analysis. Twenty-three of these 36 subjects have come to brain autopsy, and in all autopsy cases the clinical diagnosis of $\mathrm{AD}$ was confirmed pathologically.

2.2. Data Collection and Analysis. Thirty-six subjects with mild-to-moderate $\mathrm{AD}$ were evaluated by medical history, neurological and general examination, the Mini-Mental State Examination (MMSE) [10], the Clinical Dementia Rating scale (CDR) [11], the modified Hachinski ischemia score [12], and the Geriatric Depression Scale [13]. Cerebrospinal fluid and peripheral blood were collected, and brain MRI was performed.

Lumbar punctures were performed in the morning under standardized conditions at L3 to L4 or L4 to L5 interspaces, immediately aliquoted, and snap frozen at $-70^{\circ} \mathrm{C}$ until assayed. These samples had normal cell count and glucose levels, and the aliquots analyzed were matched sequentially by draw to control for CSF content drift by sequence. A CSF-to-serum ratio of albumin (CSF Albumin Index) $\geq 9.0$ was considered BBB impairment. Reproducibility of the CSF albumin index in AD over 1-year has been established (intraclass correlation coefficient $=.96$ ) [3]. Blood samples collected at the same visit as CSF were analyzed for albumin, glucose, triglycerides, total cholesterol, and high- and lowdensity lipoproteins by standardized methods performed by the Atherosclerosis Lipid Research Laboratory at Oregon Health and Science University [14]. Laboratory staff was blind to all clinical covariates.

\subsection{Statistical Analysis}

2.3.1. Descriptive Analysis. Two sample $t$-tests compared the mean differences in CSF albumin Index by subjects classified as atherogenic dyslipidemia (triglycerides $\geq 150 \mathrm{mg} / \mathrm{dL}, \mathrm{HDL}$ cholesterol $<50 \mathrm{mg} / \mathrm{dL}$ and LDL cholesterol $>100 \mathrm{mg} / \mathrm{dL}$ [15]) and metabolic dyslipidemia (the atherogenic profile without reference to LDL cholesterol) [16]. Mean differences in each lipid were compared between subjects with and without BBB impairment.

2.3.2. Primary Analysis. Multivariable linear regression models were fit to assess the relationship between lipids and CSF albumin index by including potential confounders including age, gender, ApoE-4 genotype, blood pressure, and statin use. Alpha level for significance was set at 0.05 (2tailed).

\section{Results}

Table 1 summarizes the study population. Blood-brain barrier (BBB) impairment was prevalent in $22 \%$. Frequency of 


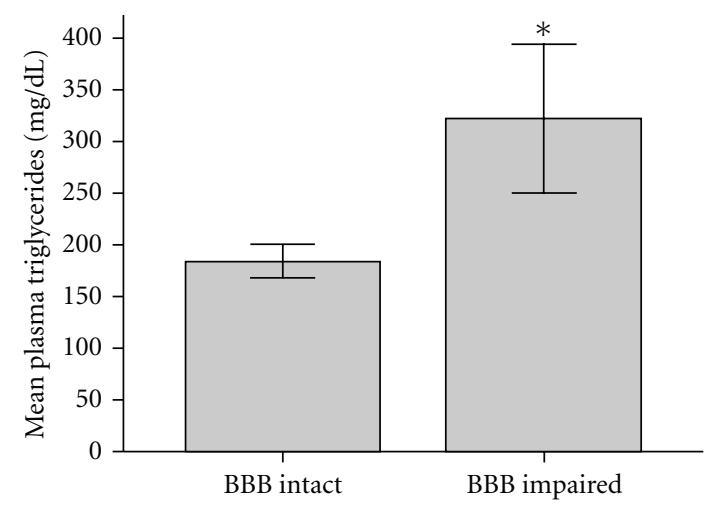

(a)

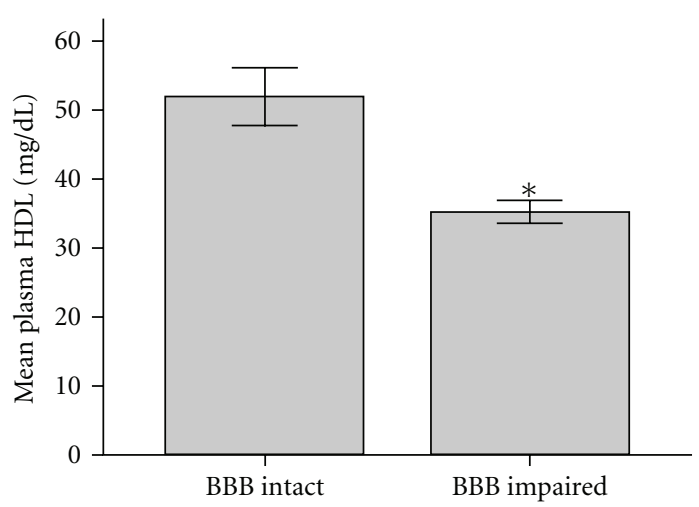

(b)

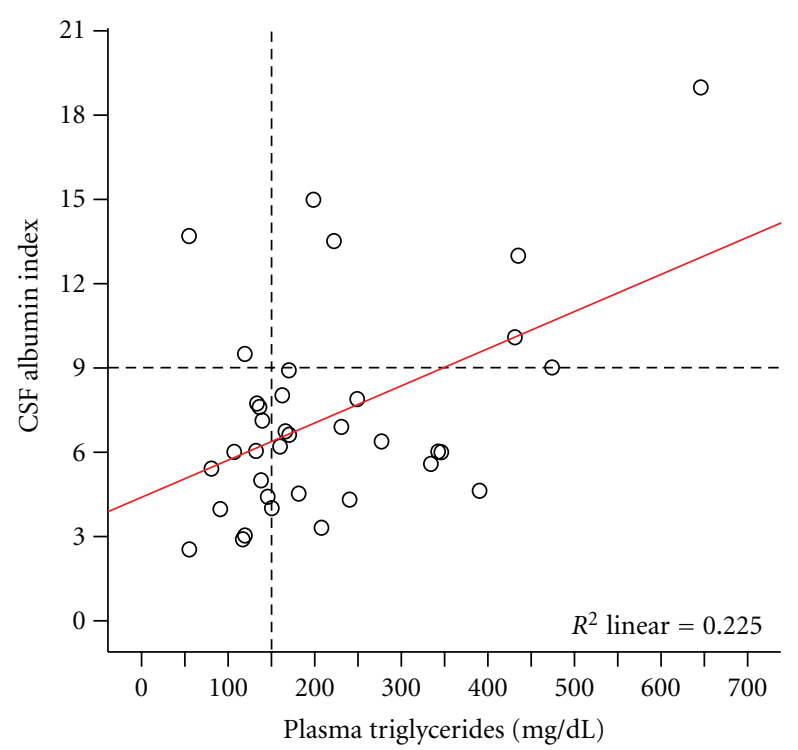

(c)

FIGURE 1: Relationship between plasma lipids and blood-brain barrier in subjects with AD. (a) Mean difference in TG $=137.89 \mathrm{mg} / \mathrm{dL}$, $P=0.007$. (b) Mean difference in HDL $=16.17 \mathrm{mg} / \mathrm{dL}, P=0.043$. Standard error bars around the mean set at $+/-1.0$. (c) Horizontal reference line separates the plots by subjects with (above) and without (below) BBB impairment. Vertical reference line indicates lipid risk threshold.

statin use was similar between the groups with and without BBB impairment $(P$ for difference $=0.251)$. Total and LDL cholesterol were not different between the two groups (total cholesterol, $P=.619$; LDL cholesterol, $P=.355$ ). However, mean plasma triglycerides were higher and HDL cholesterol lower in subjects with BBB impairment (mean difference in $\mathrm{TG}=137.89 \mathrm{mg} / \mathrm{dL}, P=0.007$; mean difference in HDL = $16.17 \mathrm{mg} / \mathrm{dL}, P=0.043$ ) (Figures $1(\mathrm{a})$ and $1(\mathrm{~b})$ ). Seventyfive percent (6/8) with BBB impairment had plasma triglycerides $\geq 150 \mathrm{mg} / \mathrm{dL}$ (range $55-646 \mathrm{mg} / \mathrm{dL}$ ) compared to $57 \%$ $(16 / 28)$ in subjects with intact BBB (range $55-389 \mathrm{mg} / \mathrm{dL}$ ). Everyone with BBB impairment had plasma HDL cholesterol below $50 \mathrm{mg} / \mathrm{dL}$ (range 29-43) compared to 60\% (17/28) in subjects with intact BBB (range 23-106 mg/dL).

\subsection{Dyslipidemia and BBB Impairment in Alzheimer's Disease.} The overall prevalence of "atherogenic" and "metabolic" dyslipidemia was $42 \%$ and $47 \%$, respectively. The prevalence of atherogenic dyslipidemia was more frequent in $\mathrm{AD}$ subjects with $\mathrm{BBB}$ impairment than in subjects without (75\% versus $32 \%$ with intact $\mathrm{BBB}, P=0.030$, Table 1$)$. Metabolic dyslipidemia was also more prevalent in BBB impaired (75\% versus $39 \%$ with intact $\mathrm{BBB}, P=0.048)$. Subjects with atherogenic dyslipidemia $(n=15)$ had mean CSF Albumin Index 2.69 units higher than those without $(n=21)(P=.029)$. Subjects with metabolic dyslipidemia $(n=17)$ had mean CSF Albumin Index 2.43 units higher than those without $(n=19)(P=0.48)$.

\subsection{Lipids Associated with BBB Integrity in Alzheimer's Dis-} ease. Linear regression analysis indicated that each $10 \mathrm{mg} / \mathrm{dL}$ increase in plasma triglyceride content was associated with a 0.13 unit increase in CSF Albumin Index $(P=0.004)$ (Figure $1(\mathrm{c})$ ). Plasma triglycerides explained $22.5 \%$ of the variation in BBB integrity. The association between plasma triglycerides and CSF Albumin Index remained significant in 
multivariable regression analysis controlled for age, gender, ApoE-4 carrier status, systolic blood pressure, and statin use $(P=0.040)$. Total cholesterol, LDL cholesterol, and HDL cholesterol were not associated with CSF Albumin Index in the regression model (data not shown).

\section{Conclusion}

These findings demonstrate a higher prevalence of dyslipidemia in Alzheimer's subjects with BBB impairment. Plasma triglycerides and HDL cholesterol were the lipids most associated with $\mathrm{BBB}$ integrity. Plasma triglycerides explain the most variation in $\mathrm{BBB}$ integrity compared to HDL, LDL, and total cholesterol.

A relationship between triglycerides and dementia has been reported in one large study [17]. This group compared the various lipid components contributing to dementia risk but did not report data on BBB integrity. To our knowledge, this is the first study to report a significant relationship between dyslipidemia and BBB integrity in Alzheimer's disease. While a causal relationship between plasma lipids and $\mathrm{BBB}$ cannot be assumed, the most plausible explanation may be that $\mathrm{BBB}$ integrity in $\mathrm{AD}$ detected by the CSF Albumin Index is related to atherosclerosis and its risk factors. For example, the omega 3 polyunsaturated fatty acids, eicosapentaenoic acid, and docosahexaenoic acid are associated with both vascular disease and dementia risk and are known to lower plasma triglyceride levels [18]. It is also plausible that the effect of dyslipidemia on the BBB is independent of atherosclerosis. This has been observed in the case of an inborn error of cholesterol metabolism with neurologic complications, cerebrotendinous xanthomatosis [19].

Although sample size in this study is a limitation, we believe that the study has particular strengths worth noting. Sixty four percent of this study population has autopsy confirmed $\mathrm{AD}$, and the entire sample had probable $\mathrm{AD}$ confirmed by consensus diagnosis. Another strength is the stability of the CSF Albumin Index as a measure of BBB integrity in living subjects [3] and the collection of fasting blood for the analysis of plasma lipids. These attributes minimize both risk of exposure and outcome misclassification and therefore strengthen the validity of the study findings in a limited sample.

The possibility that dyslipidemia is causally related to BBB impairment may be clinically significant since dyslipidemia is treatable. If this evidence is confirmed in other populations, then the next step may involve a lipid-modifying strategy to modify the natural history of AD. While it is true that statin therapy has been unsuccessful in altering the course of $\mathrm{AD}$, these current findings place emphasis on modifying triglyceride and HDL cholesterol, ideally in subjects selected on the basis of BBB impairment at baseline. Perhaps a dietary pattern or supplementation with omega-3 PUFAs and niacin would offer one strategy, since they favorably modify triglyceride and HDL cholesterol metabolism, respectively [20]. The emergence of imaging modalities for the assessment of BBB integrity will make these types of intervention more feasible.

\section{Acknowledgments}

This paper is supported by NIH/NCCAM AT004777 K23 (GLB), VA Advanced Research Development Award (JFQ), and NIH/NIA AG08017 (JAK).

\section{References}

[1] H. Link and G. Tibbling, "Principles of albumin and IgG analyses in neurological disorders. II. Relation of the concentration of the proteins in serum and cerebrospinal fluid," Scandinavian Journal of Clinical and Laboratory Investigation, vol. 37, no. 5, pp. 391-396, 1977.

[2] I. Skoog, A. Wallin, P. Fredman et al., "A population study on blood-brain barrier function in 85-year-olds: relation to Alzheimer's disease and vascular dementia," Neurology, vol. 50, no. 4, pp. 966-971, 1998.

[3] G. L. Bowman, J. A. Kaye, M. Moore, D. Waichunas, N. E. Carlson, and J. F. Quinn, "Blood-brain barrier impairment in Alzheimer disease: stability and functional significance," Neurology, vol. 68, no. 21, pp. 1809-1814, 2007.

[4] R. D. Bell and B. V. Zlokovic, "Neurovascular mechanisms and blood-brain barrier disorder in Alzheimer's disease," Acta Neuropathologica, vol. 118, no. 1, pp. 103-113, 2009.

[5] B. V. Zlokovic, An Introduction to the Blood-Brain Barrier, CRC Press, Boca Raton, Fla, USA, 1993.

[6] D. J. Begley and M. W. Brightman, "Structural and functional aspects of the blood-brain barrier," Progress in Drug Research, vol. 61, pp. 39-78, 2003.

[7] J. C. de la Torre, "Is Alzheimer's disease a neurodegenerative or a vascular disorder? Data, dogma, and dialectics," The Lancet Neurology, vol. 3, no. 3, pp. 184-190, 2004.

[8] M. Lehmann, B. Regland, K. Blennow, and C. G. Gottfries, "Vitamin B12-B6-folate treatment improves blood-brain barrier function in patients with hyperhomocysteinaemia and mild cognitive impairment," Dementia and Geriatric Cognitive Disorders, vol. 16, no. 3, pp. 145-150, 2003.

[9] L. M. Refolo, M. A. Pappolla, B. Malester et al., "Hypercholesterolemia accelerates the Alzheimer's amyloid pathology in a transgenic mouse model," Neurobiology of Disease, vol. 7, no. 4, pp. 321-331, 2000.

[10] M. F. Folstein, S. E. Folstein, and P. R. McHugh, "'Mini-mental state. A practical method for grading the cognitive state of patients for the clinician," Journal of Psychiatric Research, vol. 12, no. 3, pp. 189-198, 1975.

[11] G. Dooneief, K. Marder, M. X. Tang, and Y. Stern, “The clinical dementia rating scale: community-based validation of "profound' and “terminal' stages," Neurology, vol. 46, no. 6, pp. 1746-1749, 1996.

[12] J. T. Moroney, E. Bagiella, D. W. Desmond et al., "Metaanalysis of the Hachinski ischemic score in pathologically verified dementias," Neurology, vol. 49, no. 4, pp. 1096-1105, 1997.

[13] J. A. Yesavage, T. L. Brink, and T. L. Rose, "Development and validation of a geriatric depression screening scale: a preliminary report," Journal of Psychiatric Research, vol. 17, no. 1, pp. 37-49, 1982.

[14] Lipid Research Clinics Program, Manual of Laboratory Operations, Lipid and Lipoprotein Analysis, Edited by Resources DoHaH, National Heart, Lung and Blood Institute, Bethesda, Md, USA, 1982.

[15] "Third report of the National Cholesterol Education Program (NCEP) expert panel on detection, evaluation, and treatment 
of high blood cholesterol in adults (Adult Treatment Panel III) final report," Circulation, vol. 106, no. 25, pp. 3143-3421, 2002.

[16] S. M. Grundy, J. I. Cleeman, S. R. Daniels et al., "Diagnosis and management of the metabolic syndrome: an American Heart Association/National Heart, Lung, and Blood Institute scientific statement," Circulation, vol. 112, no. 17, pp. 27352752, 2005.

[17] C. Raffaitin, H. Gin, J. P. Empana et al., "Metabolic syndrome and risk for incident Alzheimer's disease or vascular dementia: the Three-City study," Diabetes Care, vol. 32, no. 1, pp. 169174, 2009.

[18] D. Mozaffarian and J. H. Wu, "Omega-3 fatty acids and cardiovascular disease: effects on risk factors, molecular pathways, and clinical events," Journal of the American College of Cardiology, vol. 58, no. 20, pp. 2047-2067, 2011.

[19] G. Salen, V. Berginer, and V. Shore, "Increased concentrations of cholestanol and apolipoprotein B in the cerebrospinal fluid of patients with cerebrotendinous xanthomatosis. Effect of chenodeoxycholic acid," The New England Journal of Medicine, vol. 316, no. 20, pp. 1233-1238, 1987.

[20] S. Lamon-Fava, M. R. Diffenderfer, P. H. Barrett et al., "Extended-release niacin alters the metabolism of plasma apolipoprotein (Apo) A-I and ApoB-containing lipoproteins," Arteriosclerosis, Thrombosis, and Vascular Biology, vol. 28, no. 9, pp. 1672-1678, 2008. 


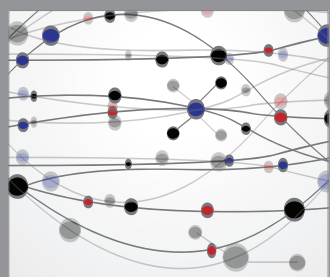

The Scientific World Journal
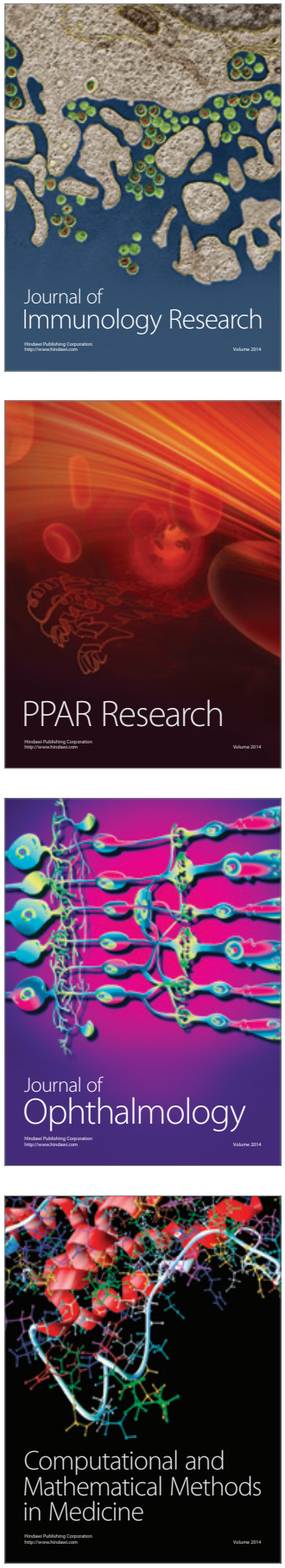

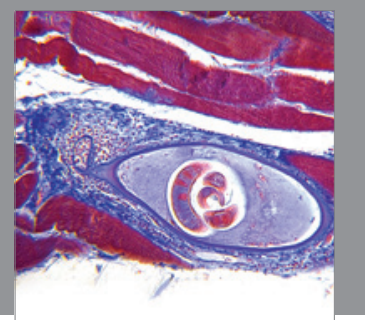

Gastroenterology

Research and Practice
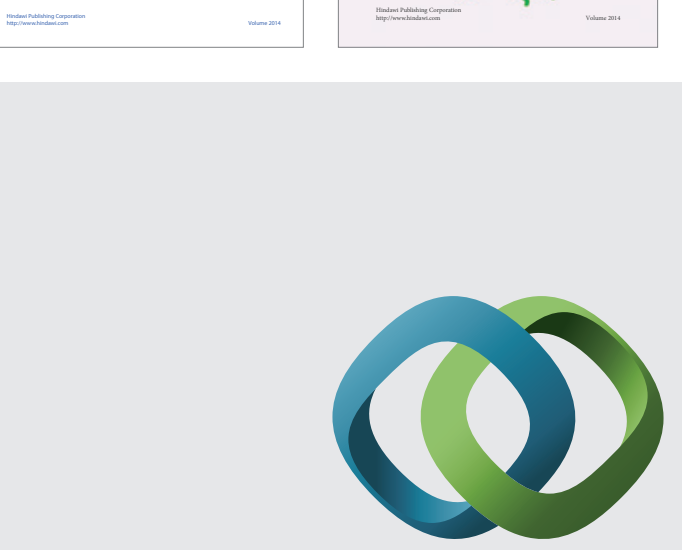

\section{Hindawi}

Submit your manuscripts at

http://www.hindawi.com
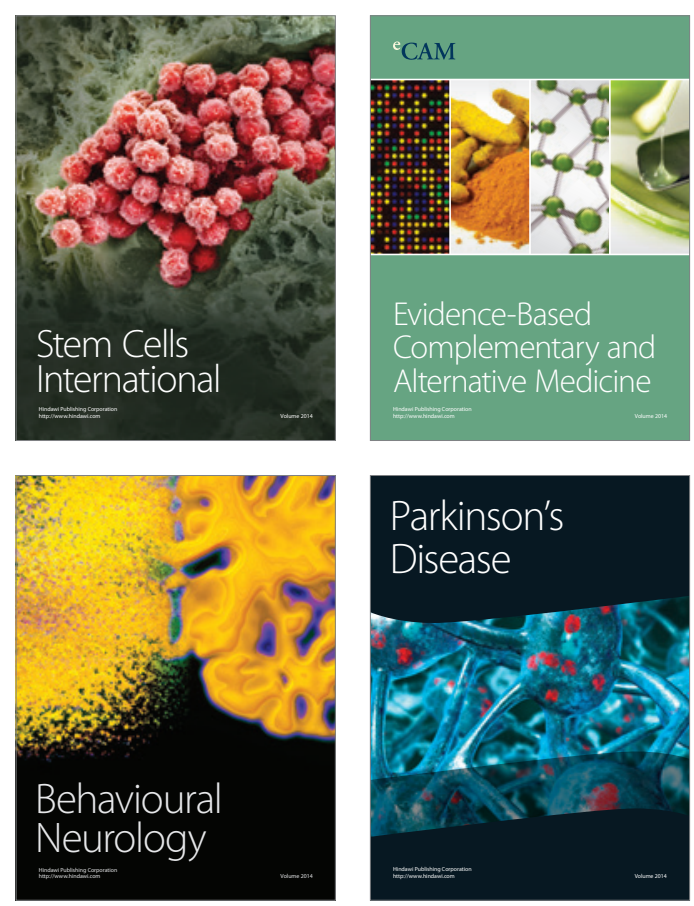

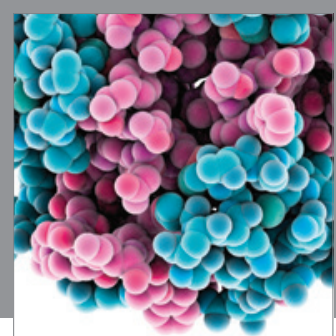

Journal of
Diabetes Research

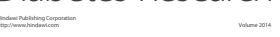

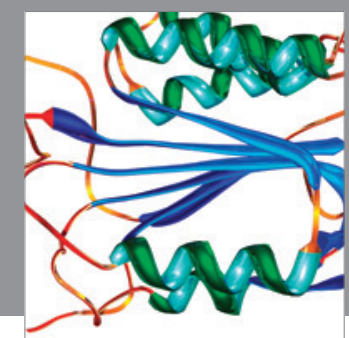

Disease Markers
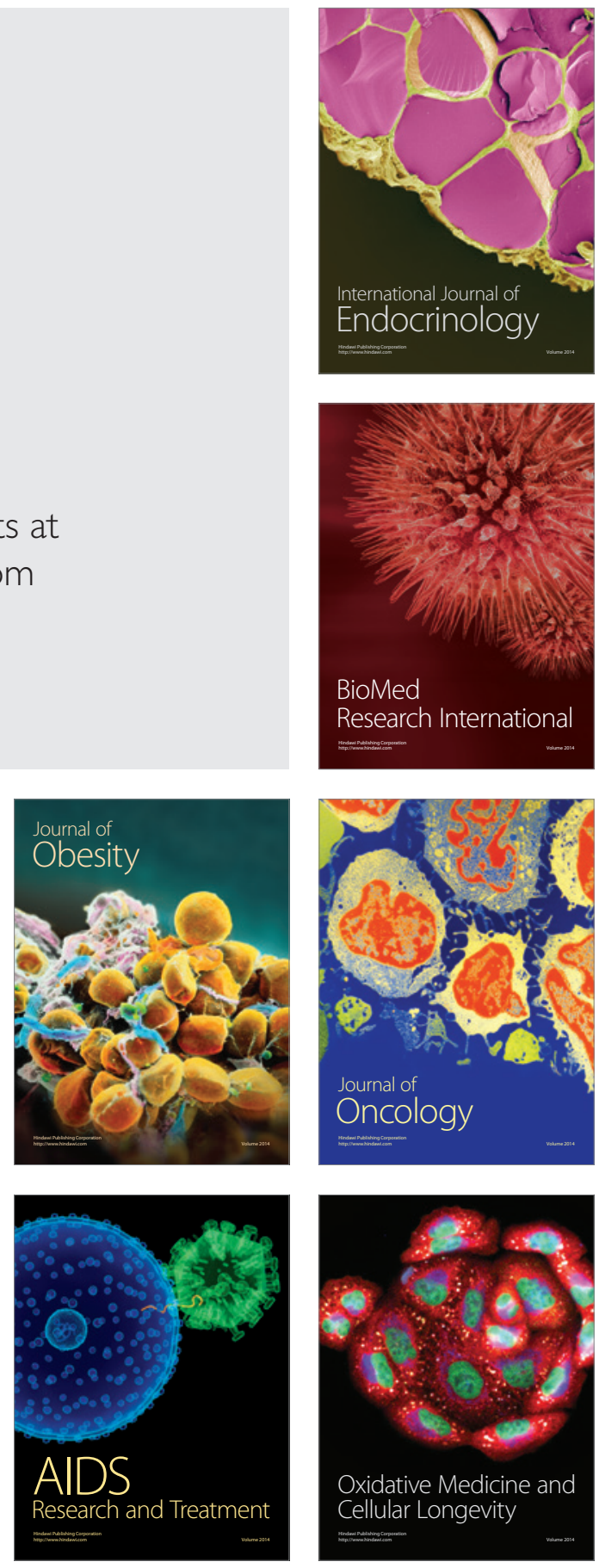